\title{
Análise da adaptabilidade e da estabilidade de genótipos de trigo como ferramenta auxiliar na recomendação de novas cultivares
}

\author{
Analysis of adaptability and stability of wheat genotypes as an auxiliary tool in recommending \\ new cultivars
}

\author{
Eduardo Caierão $^{1^{*}}$ Márcio Só e Silva ${ }^{1}$ Pedro Luiz Scheeren ${ }^{1}$ \\ Leo de Jesus Antunes Del Duca ${ }^{1}$ Alfredo do Nascimento Junior ${ }^{1}$ \\ João Leonardo Pires ${ }^{1}$
}

\section{RESUMO}

A análise da adaptabilidade e da estabilidade de genótipos de trigo auxilia o melhorista na recomendação de novas cultivares, indicando linhagens de adaptação ampla ou específica aos ambientes. Esse procedimento é particularmente importante para a cultura do trigo, principalmente levando em consideração a grande diversidade de ambientes a que o cereal é submetido no Brasil. Assim, este trabalho teve como objetivo identificar genótipos de trigo com ampla adaptação e estáveis para recomendação. Os dados para as análises foram obtidos a partir do ensaio de Valor de Cultivo e Uso (VCU) estabelecido nas diferentes regiões de adaptação do Brasil, em 2003 e 2004. O delineamento experimental usado foi o de blocos ao acaso, com 4 repetições. A variável analisada foi o rendimento de grãos. Para fins de comparação, foram usadas três cultivares como testemunhas: "CEP 24”, "BRS 194” $e$ "Ônix". Para a determinação da adaptabilidade e da estabilidade dos genótipos, foram usados os métodos descritos por Eberhart \& Russel e por Lin \& Binns. Quatro genótipos apresentaram ampla adaptabilidade e estabilidade $(\beta=1$ e $\left.S^{2} d=0\right)$ pelo método de Eberhart \& Russel destes, dois não diferiram estatisticamente da melhor testemunha. Os coeficientes de determinação foram significativos e explicaram o comportamento de todos os genótipos avaliados $\left(R^{2}>80 \% e\right.$ significativo). O modelo de Lin \& Binns gerou resultados coerentes com o de Eberhart \& Russel, para a maioria das linhagens avaliadas. Os genótipos PF 990313 e PF 980524 destacaram-se pela ampla adaptação e produtividade média, podendo ser recomendados para todas as regiões tritícolas do Brasil.

Palavras-chave: Triticum aestivum, cultivares, germoplasma, melhoramento genético.

\section{ABSTRACT}

The analysis of adaptability and stability of wheat genotypes helps the plant breeder in the recommendation of new cultivars, recommending lines of broad or specific adaptation to environments. This procedure is particularly important in wheat cropping, mainly when the great diversity of environments to which this cereal is submitted in Brazil is taken into consideration. Thus, the objective of this work was to identify broadly adapted and stable wheat genotypes for recommendation. Data for the analyses were obtained from the experiment Value of Cultivation and Use (VCU) set up in the different adaptation Brazilian regions, in 2003 and 2004. A complete randomized block experimental design with four repetitions was used. The analyzed variable was grain yield. For comparison purposes, three cultivars were used as checks: CEP 24, BRS 194, and Ônix. To determine the adaptability and the stability of genotypes, methods described by Eberhart \& Russel and by Lin \& Binns were used. Four genotypes showed broad adaptability and stability $\left(\beta=1\right.$ e $\left.S^{2} d=0\right)$ through Eberhar \& Russel's method; from these, two did not differ statistically from the best check. Determination coefficients were significant and explained the performance of all evaluated genotypes ( $R^{2}>80 \%$ and significant). Lin \& Binns model generated results coherent with Eberhart \& Russel's one in most of the evaluated lines. PF 990313 and PF 980524 genotypes performed outstandingly as to their broad adaptation and average productivity and can be recommended for all of the wheat growing regions of Brazil.

Key words: Triticum aestivum, cultivars, germplasm, genetic breeding.

\section{INTRODUÇÃO}

Somente no Rio Grande do Sul, foram recomendadas para cultivo, no ano de 2004, mais de 50 cultivares de diferentes obtentores, tornando evidente os esforços da pesquisa no desenvolvimento da cultura

'Embrapa Trigo. Rodovia BR 285, km 174, CP 451, 99001-970, Passo Fundo, RS, Brasil. E-mail: caierao@cnpt.embrapa.br. *Autor para correspondência. 
de trigo (COMISSÃO, 2004a). O sucesso comercial de uma cultivar de trigo depende, necessariamente, de seu desempenho agronômico e, portanto, o maior desafio dos melhoristas é identificar uma linhagem que apresente ótimo desempenho e estabilidade sob diferentes condições ambientais. As interações genótipos $\mathrm{x}$ ambientes trazem aos melhoristas dificuldades na identificação de linhagens superiores (KANG, 1998; JOBIM et al., 1999; JOBIM et al., 2000). A análise da adaptabilidade e da estabilidade fundamenta as decisões de um programa de melhoramento genético no momento da recomendação das cultivares em termos de regiões adaptativas (FALCONER \& MACKAY, 1998). Atualmente, muitos métodos estão disponíveis para identificar as respostas varietais aos ambientes (CRUZ \& REGAZZI, 1997). O método da análise de regressão analisa o coeficiente de regressão das características de uma cultivar em relação a um índice de ambiente (FINLAY \& WILKINSON, 1963; EBERHART \& RUSSEL, 1966). Apesar de alguns autores terem proposto modificações nesse método, o maior uso do procedimento original ainda ocorre. Embora a análise de regressão seja uma ferramenta útil para analisar a adaptabilidade, nem todos os dados se ajustam ao modelo linear e, mesmo que isso aconteça, esse modelo pode não detectar diferenças pequenas (LIN \& BINNS, 1994). Para superar essa dificuldade, foi proposto o método chamado “Análise de Superioridade”, o qual não determina nenhum modelo específico para a interação genótipo $\mathrm{x}$ ambiente (LIN \& BINNS, 1988). Duas estatísticas são calculadas: (i) o Pi, que corresponde ao valor definido como a distância média entre a cultivar em teste e o máximo; e (ii) PGEi, que é definido como um par do quadrado médio da interação entre o máximo e a cultivar testada. A cultivar é considerada superior se o valor de Pi é reduzido. Portanto, o valor de Pi é medido nas condições de todos locais e representa, de maneira geral, a adaptabilidade. A decomposição da estatística $\mathrm{Pi}$, considerando-se ambientes favoráveis e desfavoráveis, foi proposta como aprimoramento deste método (CARNEIRO, 1998). Parâmetros de estabilidade estudados em três cereais indicaram que a cultura do trigo é mais estável em rendimento de grãos quando comparada ao milho e ao sorgo (YUE et al., 1990). Interações significativas entre genótipos e ambientes têm sido descritas freqüentemente em milho (ASLAM et al., 1988) e arroz (QAYYUM et al., 2000), mas poucas informações estão disponíveis em relação a cultivares de trigo (ASIF et al., 2003).

O objetivo deste trabalho foi avaliar a adaptabilidade e a estabilidade do rendimento de grãos de genótipos de trigo do programa de melhoramento da Embrapa, para escolha das mais adaptadas e estáveis para registro e comercialização, levando em consideração duas metodologias de avaliação.

\section{MATERIAL E MÉTODOS}

Foram utilizados dados de produtividade de grãos, obtidos no ensaio de Valor de Cultivo e Uso (VCU), de sete linhagens e três cultivares de trigo estabelecidas em regiões distintas do Brasil. As linhagens, escolhidas tendo como parâmetro o desempenho superior nos ensaios de competição e a potencialidade em termos de lançamento, foram: PF 980188, PF 980537, PF 990283, PF 980524, PF 990313, PF 990404 e PF 001033. Foram utilizadas como testemunhas as cultivares “CEP 24”, “BRS 194” e “Ônix”.

Os experimentos foram conduzidos em 30 diferentes ambientes nos anos de 2003 e 2004, distribuídos em estados das regiões Sul, Sudeste e Centro-Oeste do Brasil, buscando representar todas as 12 regiões adaptativas descritas pelo Ministério da Agricultura, Pecuária e Abastecimento (MAPA) para fins de registro, conforme a tabela 1 . Para que um mesmo local de avaliação, com dois anos de ensaio VCU, fosse considerado um ambiente distinto, fundamental para a análise de adaptabilidade e estabilidade, foi realizada uma análise de variância para detectar se a interação genótipo x ambiente apresentava-se significativa. A análise foi realizada individualmente para cada local. No caso de ambientes com mais de uma época de semeadura dentro do mesmo ano de avaliação, foi observado um intervalo de 30 dias entre cada uma das semeaduas, de maneira a diferenciar bem cada uma das condições ambientais. Todos os ensaios foram estabelecidos em delineamento de blocos casualizados, com 4 repetições. A parcela experimental teve dimensão de 5,0m x 2,1m, desconsiderando as duas linhas laterais (bordaduras) para fins de avaliação. Todos os tratos culturais foram executados conforme indicação das Comissões Sul-brasileira e Centro-Sul de Pesquisa de Trigo (COMISSÃO, 2004ab). Os valores obtidos no peso das parcelas, a partir da trilha, foram corrigidos para umidade $13 \%$ e, posteriomente, transformados para $\mathrm{kg} \mathrm{ha}^{-1}$. Para as análises propostas, foram aplicados os modelos definidos pelas equações $Y_{i j}=\mu_{i}+b_{i x j}+\sigma_{i j}$, onde $Y_{i j}=$ média do genótipo $i$ no ambiente $j ; \mu_{i}=$ médiả do genótipo $i$, considerando-se todos os ambientes; $b_{i}=$ coeficiente de regressão para o genótipo $i ; x_{i}=$ índice do ambiente $j$, obtido pela média de todos os genótipos 
Tabela 1 - Locais de avaliação dos genótipos de trigo conduzidos no Rio Grande do Sul, em Santa Catarina, no Paraná e em Mato Grosso do Sul para a análise da adaptabilidade e estabilidade nos anos de 2003 e 2004.

\begin{tabular}{|c|c|c|c|c|}
\hline \multirow{2}{*}{ Estado } & \multicolumn{2}{|c|}{2003} & \multicolumn{2}{|c|}{2004} \\
\hline & Local & $\mathrm{N}^{\circ}$ Épocas & Local & $\mathrm{N}^{\circ}$ Épocas \\
\hline \multirow{5}{*}{ Rio Grande do Sul } & Passo Fundo & 1 & Passo Fundo & 2 \\
\hline & Vacaria & 1 & Vacaria & 1 \\
\hline & São Borja & 1 & São Borja & 2 \\
\hline & - & - & Santa Rosa & 2 \\
\hline & Cachoeira do Sul & 1 & Cachoeira do Sul & 2 \\
\hline \multirow{2}{*}{ Santa Catarina } & Abelardo Luz & 1 & Abelardo Luz & 1 \\
\hline & Campos Novos & 1 & Campos Novos & 1 \\
\hline \multirow{4}{*}{ Paraná } & Campo Mourão & 1 & Campo Mourão & 2 \\
\hline & Cafelândia & 1 & Cafelândia & 2 \\
\hline & Ponta Grossa & 1 & Ponta Grossa & 1 \\
\hline & - & - & Guarapuava & 1 \\
\hline \multirow{2}{*}{ Mato Grosso do Sul } & Maracajú & 1 & Maracajú & 2 \\
\hline & - & - & Ponta Porâ & 1 \\
\hline Total & & 10 & & 20 \\
\hline
\end{tabular}

no ambiente $j$, subtraída da média geral; e $\sigma_{i j}=$ desvio da regressão para o genótipo $i$ no ambiente $j$ (EBERHART \& RUSSEL, 1966) e $P_{i}=\left(\sum_{j=1}^{a}\left(X_{i j}-M_{j}\right)^{2}\right) /$ $2^{a}$, onde $P_{i}=$ estimativa do parâmetro estabilidade da cultivar $i ; Y_{i j}=$ produtividade da i-ésima cultivar no jésimo ambiente; $M_{j}=$ resposta máxima observada entre todas as cultivares no ambiente $j$; $a=$ número de ambientes (LIN \& BINNS, 1988). Utilizou-se os dois modelos de análise para obtenção de conclusões consistentes sobre o comportamento dos genótipos, já que o modelo de Linn \& Binns complementa o de Eberhart \& Russel, classificando os ambientes em favoráveis e desfavoráveis.

O Quadrado Médio do Erro (QME) da análise conjunta foi obtido pela análise de variância simples e inserido no modelo. Na execução, foi empregado o software estatístico Genes for Windows, desenvolvido pela Universidade Federal de Viçosa (CRUZ, 2001)

\section{RESULTADOS E DISCUSSÃO}

Todos os locais de experimentação, comuns aos anos 2003 e 2004, apresentaram interação genótipo $\mathrm{x}$ ambiente significativa, validando sua utilização como ambientes distintos. Dos ambientes avaliados nos dois anos, Vacaria e Santa Rosa (Época 1), em 2004, foram eliminados por apresentarem, respectivamente, coeficientes de variação elevado e problemas decorrentes de geada; assim, somente 28 foram utilizados para as análises. Apesar de os dados destes dois locais terem sido desconsiderados, outros 11 apresentaram índice ambiental negativo, ou seja, média de produtividade inferior à média de todos os locais. São eles: Abelardo Luz, Cafelândia e Maracaju, em 2003, e Santa Rosa (Época 2), São Borja (Época 2) e Campo Mourão (épocas 1 e 2), Cafelândia (época 1), Ponta Porã e Maracaju (épocas 1 e 2) em 2004. O índice de ambiente tem grande importância na interpretação dos resultados, já que dá o embasamento científico para as respostas agronômicas encontradas nas lavouras das diferentes regiões onde os ensaios estão localizados (OLIVEIRA et al., 2003). Os locais que apresentaram índice negativo caracterizam-se por serem regiões mais quentes, de menor altitude e pertencentes ao eixo Iguaçu-Missões como definição geográfica, o qual abrange o oeste dos estados do Rio Grande do Sul, de Santa Catarina, do Paraná e ainda o sul do Mato Grosso do Sul.

Não houve diferença significativa (Tukey $5 \%$ ) entre os genótipos para rendimento de grãos, sendo que nenhuma linhagem apresentou rendimento 
médio superior entre si e comparada com a melhor testemunha (“Ônix”), em valores absolutos (Tabela 2). Porém, a PF 990313 e a PF 980524 apresentaram-se altamente competitivas. Considerando que a testemunha "Ônix" é a referência atual em termos de produtividade no sul do Brasil, esses dois genótipos vão ao encontro do ideotipo desejado. A testemunha “BRS 194” foi o genótipo com menor desempenho no ensaio, com média de 3.664kg ha-1 (Tabela 2); entretanto, essa cultivar é recomendada apenas para o RS e para SC (COMISSÃO, 2004a), apresentando desempenho ruim quanto menor for a latitude, o que justifica esse comportamento em termos de média geral. A média geral de rendimento de grãos nos ambientes de avaliação não pode ser considerada como critério de adaptação, já que muitas variáveis tem interferência no comportamento dos genótipos (JOSEPHIDES, 1992).

Quatro linhagens apresentaram desempenho ideal, ou seja, adaptabilidade $(\beta=1)$ e estabilidade $\left(s^{2} d=0\right)$ nos diferentes ambientes avaliados, pelo método de Eberhart \& Russel. Em ordem decrescente, pelo desempenho de rendimento de grãos, as linhagens em questão foram: PF 990313, PF 980524, PF 980537 e PF 980188 (Tabela 2). As outras três linhagens não reuniram ambas as características, demonstrando ou adaptabilidade específica a um tipo de ambiente (favorável ou desfavorável), caso da PF 990283, ou baixa previsibilidade de comportamento, como a PF 990404 e a PF 001033. A estabilidade é fundamental para cultivares de trigo, principalmente para regiões de clima sub-tropical, onde há oscilações significativas nas condições climáticas de primavera (ASIF et al., 2003). A linhagem PF 990283 e a cultivar "BRS 194" foram os únicos tratamentos a apresentarem valor de $\beta$ diferente de 1 , sendo adaptadas a ambientes favoráveis ( $\beta$ significativamente maior que um) e desfavoráveis ( $\beta$ significativamente menor que um), respectivamente. O comportamento da PF 990283 sugere que responda bem em ambientes de temperaturas amenas e altitude elevada, mas não apresente bom desempenho quando cultivada nos locais com índice de ambiente negativo, listados anteriormente. Pelo fato de ter sido testada em 11 ambientes com esta característica, explica-se a baixa média geral do genótipo. Neste caso, sua recomendação, se lançada, deve ser dirigida para as regiões 3, 5 e 8 (altas e de temperaturas amenas) do zoneamento de Valor de Cultivo e Uso do Ministério de Agricultura, Pecuária e Abastecimento (COMISSÃO, 2004ab). O fraco desempenho da cultivar "BRS 194" também pode ser explicado por estes argumentos, porém com enfoque oposto. Por apresentar menor potencial produtivo, não responde às condições de ambiente, quando estes são favoráveis, de maneira tão positiva quanto outros genótipos, fazendo com que sua média não seja incrementada na mesma proporção (SIVAPALAN et al. 2001). Os maiores desvios ( $\mathrm{S}^{2} \mathrm{~d}$ ) foram obtidos nas cultivares "Ônix" e "CEP 24", sendo devidos à alta susceptibilidade à ferrugem da folha na primeira e ao acamamento na segunda.

Nenhuma testemunha apresentou previsibilidade de comportamento nos ambientes e nos anos avaliados (Tabela 2). O coeficiente de determinação

Tabela 2 - Desempenho médio das linhagens de trigo avaliadas quanto ao rendimento de grãos (kg ha $\left.{ }^{-1}\right)$ e estimativa dos parâmetros de adaptabilidade e de estabilidade conforme modelo de Eberhar \& Russel.

\begin{tabular}{|c|c|c|c|c|c|c|}
\hline \multirow{2}{*}{$\begin{array}{l}\text { Genótipo } \\
\text { Ônix }\end{array}$} & \multirow{2}{*}{$\begin{array}{c}\text { Média } \\
4.018 \text { ns }\end{array}$} & \multicolumn{2}{|c|}{$\beta$} & \multicolumn{2}{|c|}{$\mathrm{S}^{2} \mathrm{~d}$} & \multirow{2}{*}{$\begin{array}{c}\mathrm{R}^{2} \\
0,86\end{array}$} \\
\hline & & 1,01 & Ns & 145022 & $* *$ & \\
\hline PF 990313 & $4.017 \mathrm{~ns}$ & 1,12 & Ns & 19821 & ns & 0,95 \\
\hline PF 980524 & $4.008 \mathrm{~ns}$ & 1,03 & Ns & 85957 & ns & 0,90 \\
\hline CEP 24 & $3.938 \mathrm{~ns}$ & 0,90 & Ns & 120017 & $* *$ & 0,85 \\
\hline PF 980537 & $3.873 \mathrm{~ns}$ & 1,00 & Ns & 54452 & ns & 0,92 \\
\hline PF 990404 & 3.845 ns & 0,92 & Ns & 185464 & $* *$ & 0,81 \\
\hline PF 001033 & $3.819 \mathrm{~ns}$ & 0,95 & Ns & 127982 & $* *$ & 0,86 \\
\hline PF 980188 & $3.815 \mathrm{~ns}$ & 1,04 & Ns & 90078 & ns & 0,90 \\
\hline PF 990283 & $3.705 \mathrm{~ns}$ & 1,19 & $* *$ & 68275 & ns & 0,93 \\
\hline BRS 194 & 3.664 ns & 0,79 & $* *$ & 113009 & $*$ & 0,82 \\
\hline
\end{tabular}

$\beta=$ estimativa de adaptabilidade; $*$,** = significativamente diferente de um pelo teste $\mathrm{t}$, a $5 \%$ e $1 \%$ de probabilidade.

$\mathrm{S}^{2} \mathrm{~d}=$ estimativa de estabilidade; *,** = significativamente diferente de zero pelo teste $\mathrm{t}$, a $5 \%$ e $1 \%$ de probabilidade.

ns = não significativo

$\mathrm{R}^{2}=$ coeficiente de determinação. 
calculado pela equação de regressão explicou o comportamento de todas as linhagens e testemunhas $\left(\mathrm{R}^{2}>80 \%\right.$ significativo); destaque para PF 990313, PF 980524, PF 980537, PF 980188 e PF 990283, com coeficientes acima de $90 \%$ (Tabela 2). De maneira conjunta, os genótipos PF 990313 e PF 980524 apresentaram índices desejados em todos os parâmetros avaliados por esse método. Podem ser considerados como os melhores genótipos aqueles pertencentes ao agrupamento de beta igual a um, deslocados à direita do eixo das abscissas, com média superior à média geral dos experimentos (Figura 1). Genótipos com estas características podem ser definidos como "superiores" (SIVAPALAN et al., 2001).

A análise de Lin \& Binns revelou resultados semelhantes aos encontrados a partir do modelo de Eberhart \& Russel (Tabela 3). Considerando a expressão de comportamento, no âmbito geral, as linhagens PF 990313 e PF 980524 foram as que se destacaram, com menor valor de Pi (medida de adaptabilidade), superando até mesmo a melhor testemunha (Ônix). Por sua vez, as linhagens PF 990188 e PF 980283 foram as

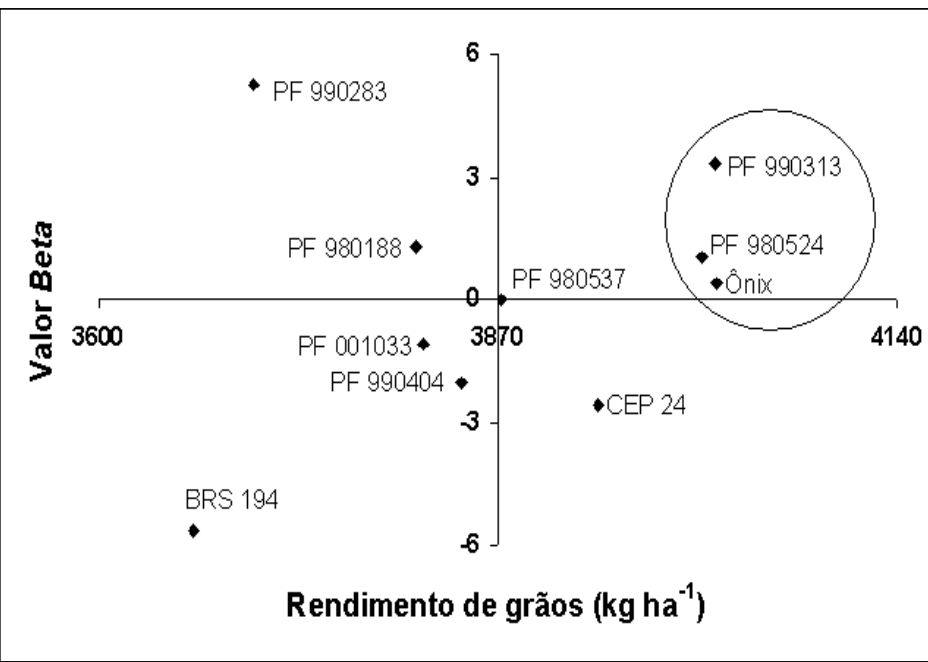

Figura 1 - Expressão gráfica do valor beta $(\beta)$ e produtividade quanto ao método de Eberhart \& Russel de diferentes cultivares/genótipos de trigo submetidos a 30 ambientes de avaliação nos anos de 2003 e 2004. Genótipos agrupados apresentaram valor $\beta$ igual e S2d igual a zero. O eixo das ordenadas representa a média geral de todos os genótipos.

dedesempenho inferior para adaptabilidade geral. Considerando ambientes favoráveis, destaque novamente para PF 980524 e PF 990313, o que pode indicar adaptação para regiões específicas. A linhagem PF 990283, definida como de alta adaptabilidade a ambientes favoráveis segundo o modelo de Eberhart \& Russel, não repetiu o desempenho segundo o modelo

Tabela 3 - Parâmetros de adaptabilidade e estabilidade da variável rendimento de grãos $\left(\mathrm{kg} \mathrm{ha}^{-1}\right)$, conforme modelo de Lin \& Binns, com direção de resposta aos ambientes avaliados.

\begin{tabular}{|c|c|c|c|c|c|c|}
\hline \multicolumn{3}{|c|}{ Comportamento geral } & \multicolumn{2}{|c|}{ Favoráveis } & \multicolumn{2}{|c|}{ Desfavoráveis } \\
\hline Genótipo & RG & $P_{i} G$ & Genótipo & $P_{i} F$ & Genótipo & $P_{i} D$ \\
\hline PF 990313 & 4.017 & 168521 & PF 980524 & 148041 & Ônix & 120475 \\
\hline PF 980524 & 4.008 & 174803 & PF 990313 & 153366 & CEP 24 & 179013 \\
\hline Ônix & 4.018 & 234691 & PF 980537 & 300152 & PF 990313 & 188340 \\
\hline PF 980537 & 3.873 & 260950 & Ônix & 322032 & PF 001033 & 208254 \\
\hline CEP 24 & 3.938 & 267230 & PF 980188 & 330099 & PF 980537 & 209687 \\
\hline PF 990404 & 3.845 & 304180 & PF 990283 & 332195 & PF 980524 & 209799 \\
\hline PF 001033 & 3.819 & 307283 & CEP 24 & 334691 & BRS 194 & 219211 \\
\hline PF 980188 & 3.815 & 323631 & PF 990404 & 359009 & PF 990404 & 232482 \\
\hline PF 990283 & 3.705 & 392059 & PF 001033 & 383011 & PF 980188 & 315172 \\
\hline BRS 194 & 3.664 & 454498 & BRS 194 & 634423 & PF 990283 & 470342 \\
\hline
\end{tabular}

$\mathrm{RG}=$ média de rendimento de grãos $\left(\mathrm{kg} \mathrm{ha}^{-1}\right)$.

$P_{i} G=$ estimativa de todos os ambientes.

$\mathrm{P}_{\mathrm{i}} \mathrm{F}=$ estimativa dos ambientes favoráveis.

$\mathrm{P}_{\mathrm{i}} \mathrm{D}=$ estimativa dos ambientes desfavoráveis. 
de Lin \& Binns. Ademais, quando avaliada quanto ao parâmetro "ambientes desfavoráveis”, foi o pior genótipo, apresentando coerência (Tabela 2).

Ambos os métodos de avaliação de adaptabilidade e estabilidade empregados foram eficientes, pois discriminaram as populações avaliadas quanto à produtividade e à capacidade de expansão nos ambientes estudados e foram coerentes entre si na maioria das interpretações.

\section{CONCLUSÕES}

As linhagens PF 990313 e PF 980524 podem ser consideradas estáveis e de ampla adaptação, podendo ser indicadas para todas as regiões produtoras de trigo do Rio Grande do Sul, de Santa Catarina, do Paraná, de São Paulo e do Mato Grosso do Sul. O genótipo PF 990283 deve ser indicado para regiões altas, acima de $600 \mathrm{~m}$, de temperaturas amenas, e para produtores que adotem alta tecnologia.

\section{AGRADECIMENTOS}

À Fundação Pró-Sementes de Apoio à Pesquisa, pela parceria na instalação, condução e colheita dos experimentos.

\section{REFERÊNCIAS}

ASIF, M. et al. Stability of wheat genotypes for grain yield under diverse rainfed ecologies of Pakistan. Asian Journal of Plant Sciences, Islamabad, v.2, n.4, p.400-402, 2003.

ASLAM, M. et al. Genotype x environment interaction and stability of performance among different varieties maize in Pakistan. Pak J Agri Res, Islamabad, v.9, p.52-55, 1988.

CARNEIRO, P.C.S. Novas metodologias de análise da adaptabilidade e estabilidade de comportamento. 1998. 168f. Tese (Doutorado em Genética e Melhoramento) Programa de Pós-graduação em Genética e Melhoramento, Universidade Federal de Viçosa.

COMISSÃO SUL-BRASILEIRA DE PESQUISA DE TRIGO E TRITICALE. Indicações técnicas da Comissão SulBrasileira de Pesquisa de Trigo e Triticale, 2004. 36.ed. Passo Fundo, 2004. 152p.

COMISSÃO SUL-BRASILEIRA DE PESQUISA DE TRIGO E TRITICALE. Informações técnicas da Comissão CentroSul Brasileira de Pesquisa de Trigo e Triticale para a safra de 2004. 20.ed. Londrina: IAPAR/Embrapa Soja, 2004. 218p. (Documentos, 01).
CRUZ, C.D. Programa genes: aplicativo computacional em genética e melhoramento. 2.ed. Viçosa: UFV, 2001. 648p.

CRUZ, C.D.; REGAZZI, A.J. Modelos biométricos aplicados ao melhoramento genético. Viçosa: UFV, 1997. 390p.

EBERHART, S.A.; RUSSEL, W.A. Stability parameters for comparing varieties. Crop Science, Madison, v.6, p.36-40, 1966.

FALCONER, D.S.; MACKAY, T.F.C. Introduction to quantitative genetics. New York: Logman, 1998. 464p.

FINLAY, K.W.; WILKINSON, G.N. The analysis of adaptation in a plant breeding programme. Australian Journal of Agricultural Research, East Melbourne, v.14, p.742-754, 1963.

JOBIM, C.I.P. et al. Análise da interação genótipo x ambiente para o rendimento de grãos em feijão. Pesquisa Agropecuária Gaúcha, Porto Alegre, v.5, p.161-171, 1999.

JOBIM, C.I.P. et al. Contribuição de variáveis ambientais à interação genótipo $\mathrm{x}$ ambiente em feijão. Pesquisa Agropecuária Gaúcha, Porto Alegre, v.6, p.27-38, 2000.

JOSEPHIDES, C.M. Analysis of adaptation of barley, triticale, durum and bread wheat under Mediterranean conditions. Eyphytica, Wageningen, v.65, n.1, p.1-8, 1992.

KANG, M.S. Using genotype-by-environment interaction for crop to cultivar development. Advances in Agronomy, San Diego, v.62, p.199-252, 1998.

LIN, C.S.; BINNS, M.R. Concepts and methods for analyzing regional trial data for cultivar and location selection. Plant Breeding Reviews, Madison, v.12, p.271-297, 1994.

LIN, C.S.; BINNS, M.R. A superiority measure of cultivar performance for cultivar x location data. Canadian Journal of Plant Science, Ottawa, v.68, n.3, p.193-198, 1988.

OLIVEIRA, A.B. et al. Emprego da análise AMMI na avaliação da estabilidade produtiva em soja. Pesquisa Agropecuária Brasileira, Brasília, v.38, n.3, p.357-364, 2003.

QAYYUM, A. et al. Evaluation of different rice genotypes for stability in yield performance. Pak J Sci Ind Res, Islamabad, v.43, p.188-190, 2000.

SIVAPALAN, S. et al. Yield performance and adaptation of some Australian and CIMMYT/ICARDA developed wheat genotypes in the West Asia North Africa (WANA) region. Australian Journal of Agricultural Research, Melbourne, v.52, p.661-670, 2001.

YUE, G.L. et al. Stability analysis of yield in maize, wheat and sorghum and its implication in breeding programme. Plant Breeding, Madison, v.104, p.72-80, 1990. 